\title{
RETHIMED JAN 161855 \\ Towards Data Fusion in Seismic Monitoring: Source Characterization of Mining Blasts with Acoustic and Seismic Records
}

\author{
Richard R. Leach, Jr. \\ Farid U. Dowla
}

July 1995

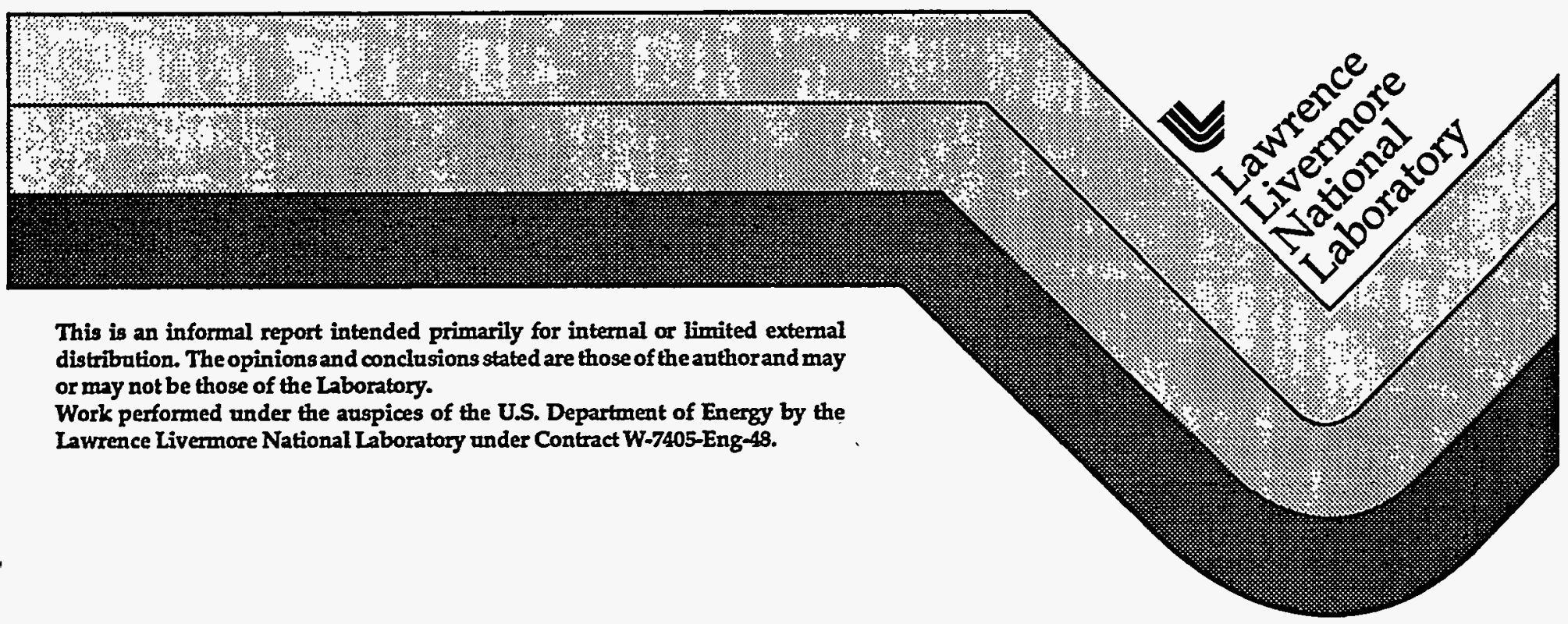




\section{DISCLAIMER}

This document was prepared as an account of work sponsored by an agency of the United States Government. Neither the United States Government nor the University of California nor any of their employees, makes any warranty, express or implied, or assumes any legal liability or responsibility for the accuracy, completeness, or usefulness of any information, apparatus, product, or process disclosed, or represents that its use would not infringe privately owned rights. Reference herein to any specific commercial product, process, or service by trade name, trademark, manufacturer, or otherwise, does not necessarily constitute or imply its endorsement, recommendation, or favoring by the United States Government or the University of California. The views and opinions of authors expressed herein do not necessarily state or reflect those of the United States Government or the University of California, and shall not be used for advertising or product endorsement purposes. 
Towards Data Fusion in Seismic Monitoring: Source Characterization

of Mining Blasts with Acoustic and Seismic Records

Richard R. Leach Jr.

Farid U. Dowla

Automated Data Processing

Research Lawrence Livermore National Laboratory

University of California, Livermore, CA 94550

July 1995 


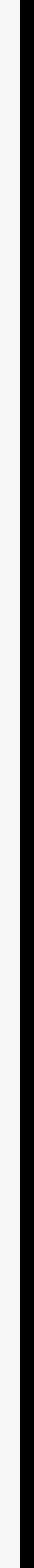




\section{Contents}

$\begin{array}{ll}\text { Abstract } & 3\end{array}$

Introduction $\quad 4$

Experimental Data and Observations 5

$\begin{array}{ll}\text { Recommendations for Data Fusion Analysis } & 7\end{array}$

$\begin{array}{ll}\text { Summary } & 8\end{array}$

$\begin{array}{ll}\text { Acknowledgments } & 9\end{array}$

References 9

$\begin{array}{ll}\text { Figure Captions } & 9\end{array}$

Figures 


\title{
Towards Data Fusion in Seismic Monitoring: Source Characterization of Mining Blasts with Acoustic and Seismic Records
}

\author{
Richard R. Leach Jr. \\ Farid U. Dowla
}

\begin{abstract}
Event identification that combines data from a diverse range of sensor types, such as seismic, hydroacoustic, infrasound, optical, or acoustic sensors, has been discussed recently as a way to improve treaty monitoring technology, especially for a Comprehensive Test Ban Treaty. In this exploratory study we compare features in acoustic and seismic data from ripple-fired mining blasts, in an effort to understand the issues of incorporating data fusion into seismic monitoring. We study the possibility of identifying features such as spectral scalloping at high frequencies using acoustic signals recorded in the near field during mining blasts. Recorded acoustic and seismic data from two mining blasts at Carlin, Nevada, were analyzed. Seismic accelerometer signals were recorded from 0.2 to $250 \mathrm{~Hz}$ at distances of 2.6 and $3.9 \mathrm{~km}$. The mining blasts studied in this report have well-characterized firing patterns. We have found that there is a clear presence of the periodic and impulsive nature of the ripple-fire source present in the acoustic recordings at high frequencies. We have discovered that the arrival time and duration of the acoustic recordings are also clearly discernible at high frequencies. This is in contrast to the absence of these features in seismic signals, due to attenuation and scattering at high frequencies. Acoustic signals were digitized with a sample rate of 30 $\mathrm{kHz}$ from the audio track of an 8-mm video camera near each blasting site. The association of signals from different sensors offers solutions for difficult monitoring problems. Seismic or acoustic signals individually may not be able to detect a nuclear test hidden under a typical mining blast. However, the presence of an underground nuclear test during a mining event could be determined by deriving the mining explosion source from the acoustic recording, modeling a seismic signal from the derived source, and subtracting the modeled seismic signal from the seismic recording for the event. Recommendations in the design of data fusion systems for treaty monitoring are suggested.
\end{abstract}




\section{Toward Data Fusion in Seismic Monitoring: \\ Source Characterization of Mining Blasts with Acoustic and Seismic Records}

\section{Introduction}

To improve our ability to verify treaties limiting or banning nuclear tests, we may need to combine seismic data with data from other types of sensors. The terms "data fusion" or "synergy" have been used recently in treaty verification discussions. The technical aspects of monitoring all forms of seismic activities and providing effective discrimination systems are complex and challenging, and it is generally acknowledged that present treaty monitoring technology needs to be improved.

One area of concern is the threat of clandestine nuclear testing under the guise of a normally operating mine. For example, an inability to detect a nuclear test hidden under a typical mining blast would constitute a serious problem in treaty monitoring. Typical mining operations can consist of a series of explosions separated from each other by 15 to $100 \mathrm{~ms}$. The main utility of this type of firing pattern is to optimize the rock breakage while minimizing seismic damage. Seismic monitoring at regional distances of such explosion patterns might allow such recordings to be classified as a mining event. Recorded seismic time-history data of a typical mining event are illustrated in Figure 1a. The blast in this case was located $2.6 \mathrm{~km}$ from a seismometer and consisted of a series of 34 subevents, $65 \mathrm{~ms}$ apart. The seismometer that recorded the signals analyzed in this report is a Wilcoxon 731 low-noise accelerometer flat to acceleration between 0.2 and $1000 \mathrm{~Hz}$, with a 6-pole filter low-passed at $250 \mathrm{~Hz}$. Because the seismometer was located at the surface, the later arriving acoustic wave recorded by the seismometer is seen clearly after high-pass filtering the signal (Figure 1c). We discuss the characteristics of this air-wave arrival in the next section.

Seismic spectrogram analysis often works quite well in classifying mining events. However, there are instances for which this method becomes inadequate. One example is when the ripple-fire time-delayed detonations occur very close to each other (i.e., when mining operations use very short delays which are on the order of $15 \mathrm{~ms}$ ). These short firing delays result in higher frequencies at which spectral scalloping is observed. Because high frequency signals are strongly attenuated, it becomes more and more difficult to identify the scalloping in the spectra of the recorded seismic data. Depending on the geology, significant high frequency attenuation may occur even at close distances.

Another example is when mining operations experiment with firing patterns that have variable firing delays. For these explosions, the area to be blasted is divided into sections with different firing delays. Instead of the normal evenly spaced sequence of subexplosions, there are regions with different firing delays that occur simultaneously, resulting in a complicated source signal consisting of many superimposed firing patterns.

A potentially powerful approach to resolve these issues is to supplement seismic 
monitoring with acoustic analysis of mining blasts. Acoustic signals might be more noisy at low frequencies; for example, noise induced by adverse weather conditions such as wind and storms often generate low frequency noise. However, acoustic signals do not suffer from the strong attenuation of high frequency propagation of seismic signals. Combining both acoustic and seismic signals could improve the monitoring capability at a suspect mine. In addition, the cost of complementing seismic with acoustic monitoring is relatively low.

\section{Experimental Data and Observations}

We examined seismic and acoustic signals recorded from two mining events that occurred in the Newmont Gold Company (NGC) gold quarry near Carlin, Nevada, in April 1995. A map of the quarry is shown in Figure 2a where M1 indicates the location of the first mining explosion or event, $\mathrm{M} 2$ indicates the location of the second mining event. Microphone locations are denoted by $\mathrm{P} 1$ and $\mathrm{P} 2$. Seismic signals used in this study were recorded by two seismometers denoted by B3 and B4. The B3 seismometer is located at distances of 3.9 and $2.6 \mathrm{~km}$ from M1 and M2, respectively. The B4 seismometer is located at distances of 0.9 and $1.7 \mathrm{~km}$ from M1 and M2, respectively. The general topography of the region is shown as well as several shaded regions indicating the working levels in the main Gold Quarry Pit, which are at 4850-, 5350-, and 5600-ft elevations (above sea level). M2 was located in the bottom of the main Gold Quarry Pit and M1 was located at the more shallow Tusk Pit. An example firing pattern is shown in Figure $2 b$.

Event M1 occurred on April 14 and consisted of a series of 34 rows of explosions. Each row contained from 20 to 30 individual explosions that were detonated in two equal halves separated by a 17 -ms delay. The 10 to 15 individual explosions in the halfrows were detonated simultaneously. There was a 50-ms delay between rows. The second event, M2, occurred on April 20 and consisted of a series of 22 rows of explosions. Each row contained from 2 to 9 individual explosions that were detonated simultaneously. The delay between detonation from one row to the next was $65 \mathrm{~ms}$.

The acoustic signals were recorded on the audio track of a Sony $8 \mathrm{~mm}$ video camera at a line-of-sight distance of approximately $0.3 \mathrm{~km}$ for both the M1 and M2 event. These acoustic signals were digitized with a sample rate of $30 \mathrm{kHz}$ and were stored on computer disk. Figure 3a shows the raw (unfiltered) time-series explosion recording from the M1 event. Two seconds of pre-event background noise were cut from the raw data (shown in red), and two seconds of explosion signal were cut from the raw data (shown in green). The Fourier transform amplitude of each of the two cut-out signals is shown superimposed in Figure $3 \mathrm{~b}$ (background noise in red and explosion signal in green). Because of the bandpass limitations of the recording equipment, the explosion signal appears from about 200 to $5000 \mathrm{~Hz}$. In this band, the explosion signal clearly has a high signal-to-noise (SNR) ratio. A spectrogram of the acoustic signal should show a clear signature at these frequencies.

Figures 4 and 5 contain the recorded time history data and spectrogram results for mining events $\mathrm{M} 1$ and $\mathrm{M} 2$, respectively. All time series signals in these two figures were bandpass filtered with a fourth order Butterworth filter. The seismic signals shown were recorded at the B3 seismic station. The limits of the seismic signal were from 1 to $100 \mathrm{~Hz}$. The limits of the acoustic signal were from 500 to $1000 \mathrm{~Hz}$. 
The seismic signals show spectral scalloping for M1 in Figure 4a as a series of horizontal ridges at 20 and $40 \mathrm{~Hz}$ in the spectrogram. This corresponds to the $50-\mathrm{ms}$ firing delay of M1. Figure 5a for M2 shows scalloping at 15, 30, 45, and $60 \mathrm{~Hz}$, which corresponds to the 65-ms firing delay of M2. In contrast, the spectral scalloping is much more evident in the acoustic spectrograms of Figures $4 \mathrm{~b}$ and $5 \mathrm{~b}$. Clear presence of spectral scalloping for the acoustic signals is seen up to $5000 \mathrm{~Hz}$.

Figure $2 b$ illustrates an example of a firing pattern in which rows of explosions are detonated with a fixed delay between the rows. The firing pattern of M2 is a similar, simple firing pattern. A spectral analysis of events such as M2 reveal spectral peaks at the fundamental frequency of the firing rate and subsequent harmonics. The firing pattern from M1, however, is a more complex pattern composed of two superimposed firing patterns that produce a weaker seismic spectrogram pattern. The total mining event M1 has a duration of approximately $2.2 \mathrm{~s}$, whereas M2 has a duration of approximately $1.4 \mathrm{~s}$. In addition, M1 has a longer propagation path than $\mathrm{M} 2$ (see Figure 2a). For seismic signals, the longer the propagation path, the more the higher frequencies will be affected by the Earth's natural attenuation properties.

The explosion's airwave is the later arriving seismic signal in Figure 1c. The travel path of this acoustic signal is from the mining blasts, up $0.25 \mathrm{~km}$ to the brim of the Gold Quarry pit, and $2.6 \mathrm{~km}$ to the seismic sensor. Distortion is introduced by the reflections off the walls of the pit as the acoustic wave propagates up and out of the pit and toward the seismic sensor. The resulting arrival somewhat resembles a typical seismic event that starts out strong, then attenuates quickly, only to build up again for the duration of the event. This illustrates why it might be difficult to distinguish a normal air-wave mining blast arrival from one masking a test event.

Ideally, sensors used to record acoustic energy should be positioned in an optimal line-of-sight orientation with the source. In some cases, a reasonably clear acoustic path allows seismic sensors placed on the surface to record the later arriving air-wave as was seen in Figure 1c for M2. However, using a seismic sensor to record acoustic waves is often unsuccessful due to poor coupling because of path obstructions. Seismic sensors are designed and positioned for optimal coupling and recording of ground motion and without regard to their receptivity to air-waves. For example, in Figure 6, M1 recorded at B3 is shown. The acoustic arrival is rather weak, probably due to the positioning of the seismic sensor and the topography of the region. Figure 2a shows several rises in the topography between the source and B3.

The air-wave arrival from M2 was cut from the seismic signal recorded at B3 and passed through a fourth order Butterworth 50 to $250 \mathrm{~Hz}$ bandpass filter. The filtered signal and the resulting spectrogram are shown in Figure 7. With respect to duration and spectral signature over the entire filtered band of frequencies, these results are similar to the acoustic signal shown in Figure $5 b$ for the same explosion. The acoustic signal from both the microphone and seismometer offer a clear duration signature. They also have the characteristic horizontal, evenly spaced 'ridges' at the higher frequencies.

The seismic air-wave arrival, however, has a potential for complicating the seismic data; particularly for long-duration blasts. In close-in monitoring scenarios, the air-wave could arrive during the seismic signal. The resulting signal would be a combination of the emerging seismic signal and the arriving acoustic signal. Measuring the duration of the event would be hampered by the distortion in the coda resulting 
from the air-wave arrival.

As stated earlier, another potential problem is the threat of masking a nuclear event by deliberately detonating a nuclear event coincidentally with a mining blast. This is a very complex and difficult problem to solve. The use of both acoustic and seismic waves may help us to identify these "hide-in" quarry blast/nuclear explosions. For example, acoustic sensors in this scenario would primarily record the energy from the mining blast due to poor acoustic coupling of a deeply buried, low yield underground nuclear explosion. The mining blast source could be derived from this acoustic signal and used to model an "mining explosion only" seismic signal which could then be subtracted from the recorded seismic signal. Since good coupling allows the seismic sensor to record ground motion resulting from both the mining blast and the nuclear explosion, the residual should reveal the presence of a nuclear explosion signal.

Discrimination may be easier with the combination of acoustic and seismic data because the later arriving airwaves in the acoustic signal are absent. Accurate measurement of source duration is necessary for proper identification of mining events. At reasonable monitoring distances, the coda of a seismic signal makes it difficult to discern the end of the event. Figures $4 b, 5 b$, and 7 illustrate the relative ease of using an acoustic spectrogram to measure the duration of the two mining events in contrast to the seismic spectrograms.

Seismic signals propagate better at lower frequencies due to the natural attenuation and scattering properties of the Earth. The acoustic signals are more stable and, for instance, offer a clear measure of the duration of the event over a broader range and at higher frequencies. In addition, the seismic signals are more subject to distortion due to path effects. The acoustic signal could in most cases be optimized for a clear lineof-sight path, whereas the seismic path is highly variable and difficult to characterize. Monitoring capabilities could be optimized by combining seismic signal stability at low frequencies with acoustic signal stability at higher frequencies for improved source characterization. A final observation is that the individual explosions should ideally produce a distinct temporal signature in the time-series waveforms. Figures 4 and 5 show that the acoustic signals may have a clear advantage in discerning the distinct pulses in the waveform. Short-distance acoustic propagation paths will not have highfrequency attenuation and scattering problems normally associated with seismic signals. However, acoustic sites should be chosen carefully to avoid multi-pathing (echoing).

\section{Recommendations for Data Fusion Analysis}

Each type of signal, seismic and acoustic, has clear advantages and disadvantages when used to identify nuclear and non-nuclear sources. Seismic data have the advantage at very long distances. On the other hand, initial observations indicate that the combination of acoustic information with seismic at close-in distances can strengthen monitoring capabilities at a particular mine. We need to further study the limitations of these two signal types and focus on characterizing a distinct frequency signature in the recorded signals. A recent AFTAC Seismic Review Panel meeting noted (Herrin 1995) that a seismic array at Lajitas is capable of recording air-wave signals from quarry blasts in Mexico. In fact, the Lajitas array detected an air-wave 16 minutes after the P wave, thus identifying the event as a surface explosion. This indicates that air-waves could be 
useful in resolving event type at regional distances. Regional acoustic data would probably be detected and recorded as infrasonic data.

In the following, we summarize some of the technical issues that need to be addressed and resolved before acoustic and seismic data can be used jointly to improve verification capabilities. We recommend conducting field experiments and doing a literature review to address these issues.

(i) Empirically, how do the propagation and attenuation characteristics of acoustic signals vary with distance (near-field) and frequency? Field experiments can be performed to collect the necessary data in order to address this issue.

(ii) How accurately can mining explosion source functions be characterized from acoustic recordings? The acoustic signal will often perform better than a seismic recording for source characterization as in the case of very short delays between mining blasts.

(iii) At what distances and where should acoustic sensors be located to monitor mining operations? High-quality acoustic sensors, or an acoustic array of sensors can be used to form beams to reduce background noise, and might thus allow obtaining a high-resolution signature of explosion sources. An acoustic array could also be used to reduce ambient low-frequency acoustic noise. Acoustic signal coherence (spatial) as a function of frequency must be documented for proper design of acoustic arrays.

(iv) What type of equipment will be needed to implement data fusion systems? We observed that a combination of the two types of sensors would be useful in validating source characteristics. (Sensors we used in this study compliment each other: seismic sensors were good for low frequencies, and the acoustic sensors were good at higher frequencies). Finally, high-speed video or photo-diode sensors could potentially strengthen a monitoring system's source characterization capabilities. We need to understand and document the specifications (capabilities and limitations) of acoustic sensors and pressure transducers.

\section{Summary}

To understand the issues of data fusion, we looked at seismic and acoustic data from the same mining blasts. Even with a very poor quality acoustic sensor data (recorded accidentally), we saw that the spectral scalloping effects are clearly present in the high frequencies. This is significant because, for complex firing patterns and for short time delays, acoustic signatures can be a very useful way to characterize source features such as duration and ripple-firing.

Data fusion should not be viewed as just a "bonus" to a standard approach. A monitoring site fitted with appropriately designed sensors can be significantly more powerful than the same site equipped with only one type of sensor. We have shown in this report that mining duration measurement, source characterization, and discrimination may be greatly improved by combining two types of sensors.

Data fusion techniques will be more costly with the addition of high quality sensors as well as arrays of sensors. It may be more practical to use data fusion technology in portable systems, or systems located in high-risk monitoring regions. 
However, in critical monitoring situations, data fusion may be the only approach that can reliably detect clandestine nuclear testing. The addition of acoustic sensors at seismic stations for monitoring mining blasts is an important step toward the ultimate realization of improved treaty verification technology using data fusion techniques.

\section{Acknowledgments}

We thank Keith Nakanishi for the comment that led to this study, Jim Hannon, Paul Kasameyer, Howard Patton, and Jay Zucca for many helpful comments that improved this manuscript significantly, Steve Jarpe, Don Rock, and Steve Hunter for providing us with the data and valuable discussions, and Elaine Price for her editorial help. This work was performed under the auspices of the U.S. Department of Energy by Lawrence Livermore National Laboratory under contract W-7405-Eng-48.

\section{References}

Baumgardt, D. R., and K. A. Ziegler (1988). Spectral evidence for multiplicity in explosions: application to regional discrimination of earthquakes and explosions, Bull. Seism. Soc. Am., 78, 1773-1795.

Hedlin, M. A. H., J. B. Minster, and J. A. Orcutt (1989). The time and time-frequency characteristics of quarry blasts and chemical explosions recorded in Kazakhastan, U.S.S.R., Geophys. J., 99, 109-122.

Herrin, E. (1995). Presentation at the AFTAC Seismic Review Panel.

Smith, A. T. (1993). Discrimination of explosions from simultaneous mining blasts, Bull. Seism. Soc. Am., 83, 160-179.

\section{Figure Captions}

Figure 1. Acceleration signal (vertical) of mining event (M2) located at the NGC gold quarry near Carlin, Nevada. The seismometer (B3) used to record the signals in this report was the Wilcoxon 731 low-noise accelerometer, flat to acceleration between 0.2 and $1000 \mathrm{~Hz}$ and recorded with a 6-pole filter low-passed at $250 \mathrm{~Hz}$. Raw signal and filtering effects are shown. (a). Explosion arrival and the later arriving acoustic air-wave are marked. Filtering results are shown in (b) and (c). A fourth-order Butterworth filter was used with a corner frequency of $50 \mathrm{~Hz}$ for a low-pass filter in (b) and a high-pass filter in (c).

Figure 2. Map of the mining events recorded at the NGC gold quarry (left) showing the location of the April 14, 1995, explosion (M1) and the April 20, 1995, explosion (M2). Acoustic signals were recorded at P1 and P2 at a distance of about $0.3 \mathrm{~km}$ for events M1 and M2, respectively. Seismic signals were recorded at B3 for M1 and M2 at distances of 2.6 and $3.9 \mathrm{~km}$. The green shaded regions are the working levels in the Gold Quarry pit, which are at 4850-, 5350-, and 5600-ft elevations (above sea level). The dark green lines 
are local roads. An example (not M1 or M2) firing pattern (right) shows the rows (dotted lines) as well as the individual explosion sites (" $\left.+s^{\prime \prime}\right)$. Delays between row detonations were approximately $50 \mathrm{~ms}$ for M1 and $65 \mathrm{~ms}$ for M2. Hole spacing for the individual explosions were $16 \times 16 \mathrm{ft}$ for $\mathrm{M} 1$ and $18 \times 18 \mathrm{ft}$ for $\mathrm{M} 2$. Individual salvos were approximately 0.10 ton.

Figure 3. Raw (unfiltered) time-series explosion recording from the M1 event (a). Two seconds of pre-event background noise were cut from the raw data (shown in red), and two seconds of explosion signal were cut from the raw data (shown in green). The fast Fourier transform (FFT) magnitude of each of the two cut-out signals is shown superimposed in Figure $3 \mathrm{~b}$ (background noise in red and explosion signal in green). Because of the bandpass limitations of the recording equipment, the explosion signal appears from about 200 to $5000 \mathrm{~Hz}$.

Figure 4. $\quad$ M1 mining explosion event recorded at B3 and P1 showing filtered time series and resulting spectrogram for seismic signal (a) and for acoustic signal (b). All time series signals were bandpass filtered with a fourth order Butterworth filter. The seismic signal was bandpass limited from 1 to $100 \mathrm{~Hz}$. The acoustic signal was bandpass limited from 500 to $1000 \mathrm{~Hz}$.

Figure 5. $\quad \mathrm{M} 2$ mining explosion event recorded at B3 and P2 showing time series and resulting spectrogram for seismic signal (a) and for acoustic signal (b). All time series signals were bandpass filtered with a fourth order Butterworth filter. The seismic signal was bandpass limited from 1 to $100 \mathrm{~Hz}$. The acoustic signal was bandpass limited from 500 to $1000 \mathrm{~Hz}$.

Figure 6. Acceleration signal (vertical) of mining event (M2) located at the NGC gold quarry near Carlin, Nevada. The seismometer (B3) used to record the signals in this report was the Wilcoxon 731 low-noise accelerometer, flat to acceleration between 0.2 and $1000 \mathrm{~Hz}$ and recorded with a 6-pole filter low-passed at $250 \mathrm{~Hz}$. Raw signal and filtering effects are shown. (a). Explosion arrival and the later arriving acoustic air-wave are marked. Filtering results are shown in (b) and (c). A fourth-order Butterworth filter was used with a corner frequency of $50 \mathrm{~Hz}$ for a low-pass filter in (b) and a high-pass filter in (c).

Figure 7. The air-wave was cut out of the seismic signal recorded at B3 from explosion M2 and passed through a fourth order Butterworth 50 to $250 \mathrm{~Hz}$ filter. The filtered signal (top) and the resulting spectrogram (bottom) are shown. The duration of the explosion and the spectral scalloping signature for the first arrival are consistent for the entire band and show a striking similarity to the acoustic spectrogram from the video camera (Figure 5b) 

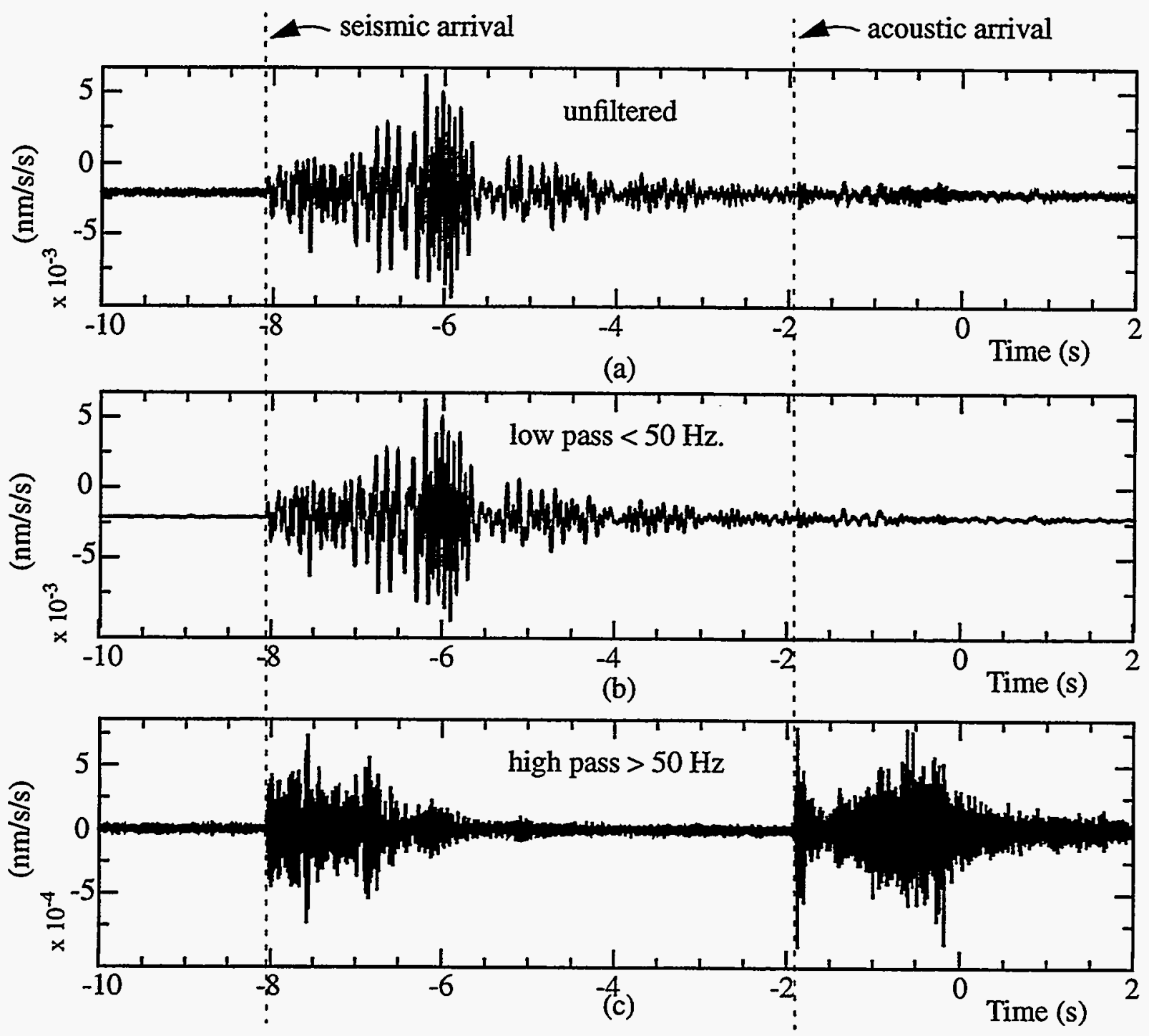

Figure 1 


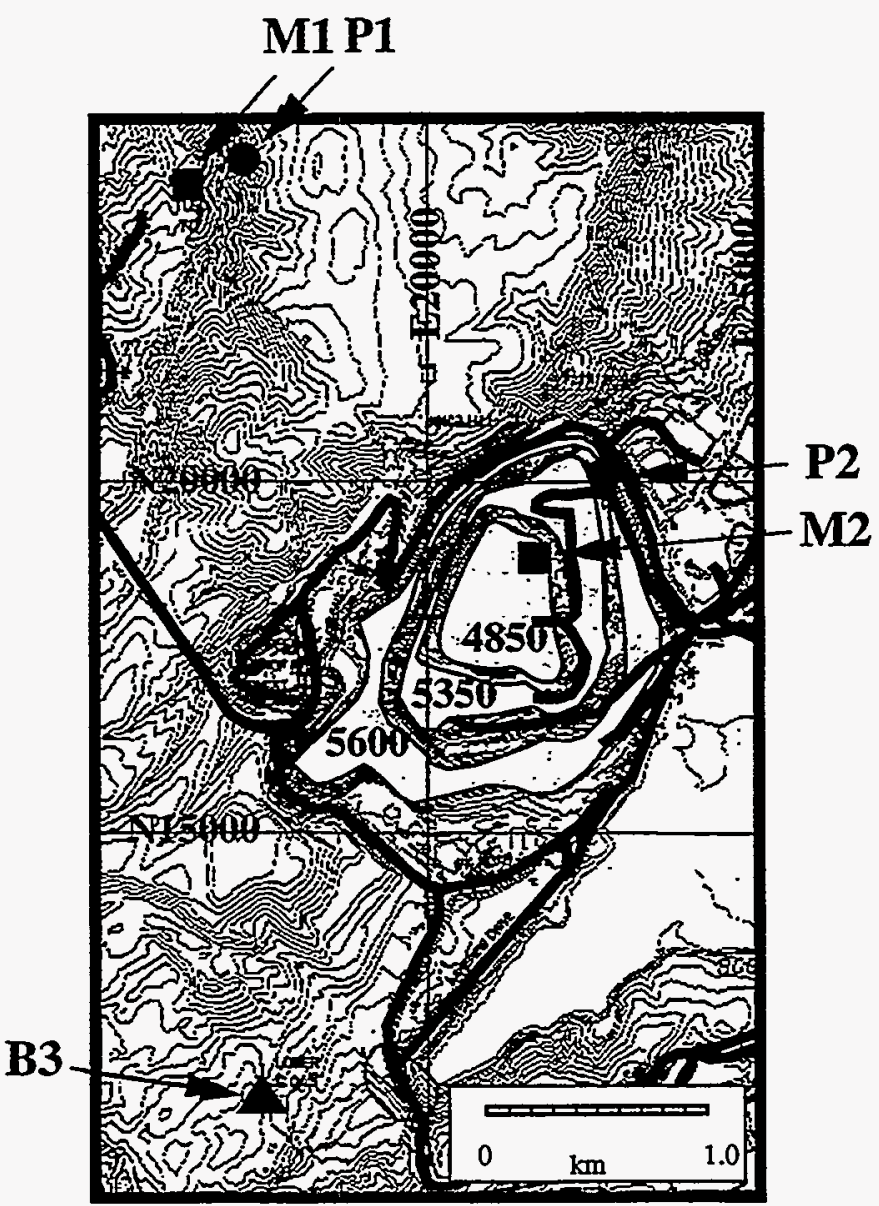

CENTER $40.79-116.21 \quad 0.0$ PROJECTION LAMBERT, WINDOW CORNERS

$40.7642 \quad-116.2425 \quad 40.8082 \quad-116.1951$

(a)

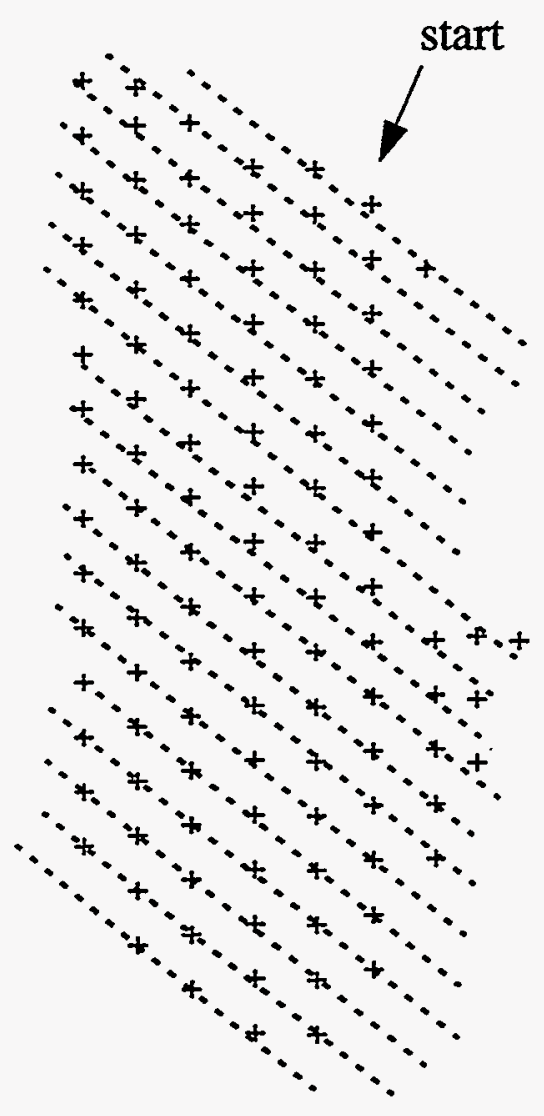

Firing pattern example

(b)

Figure 2 
Explosion 1 -acoustic
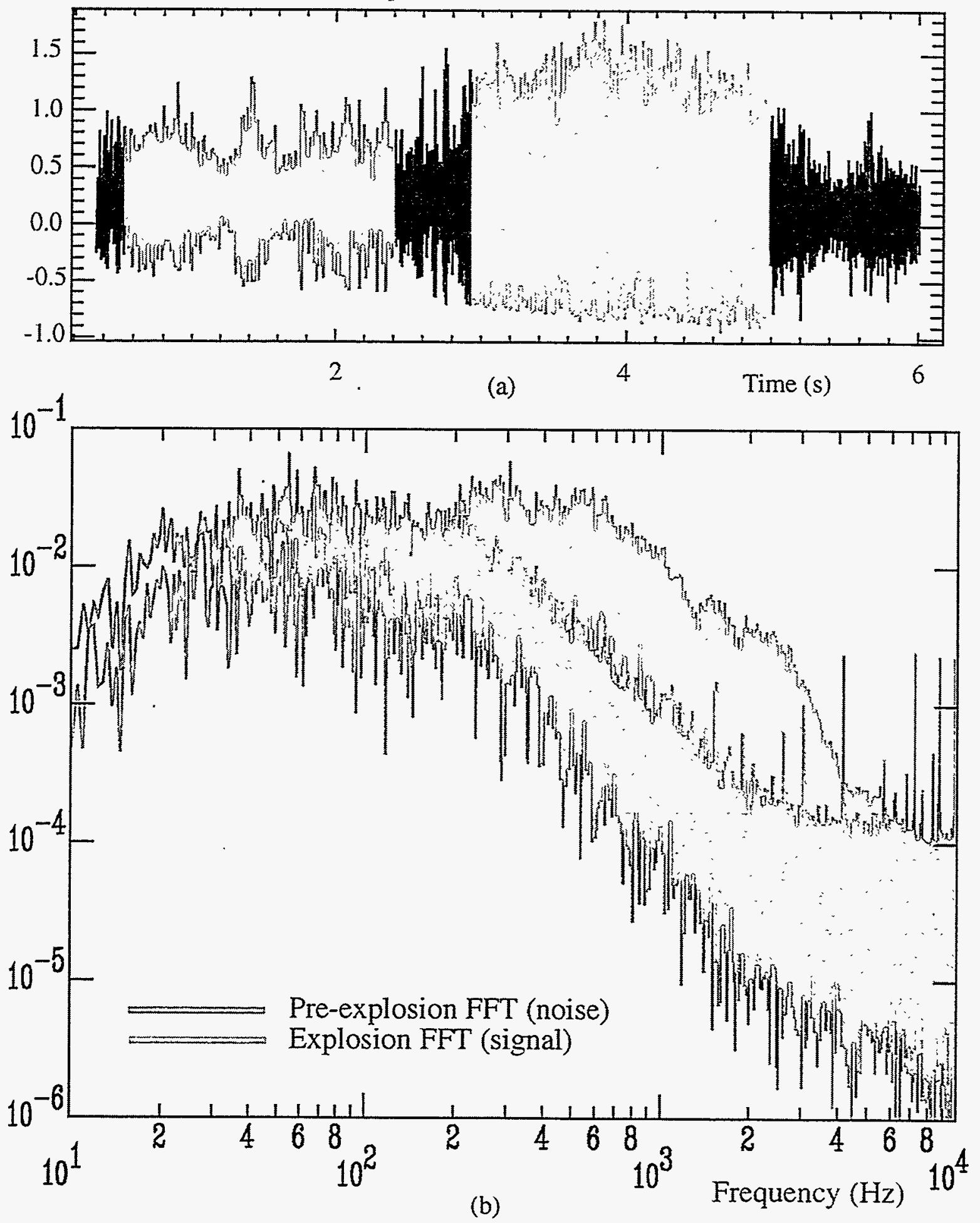

Figure 3 


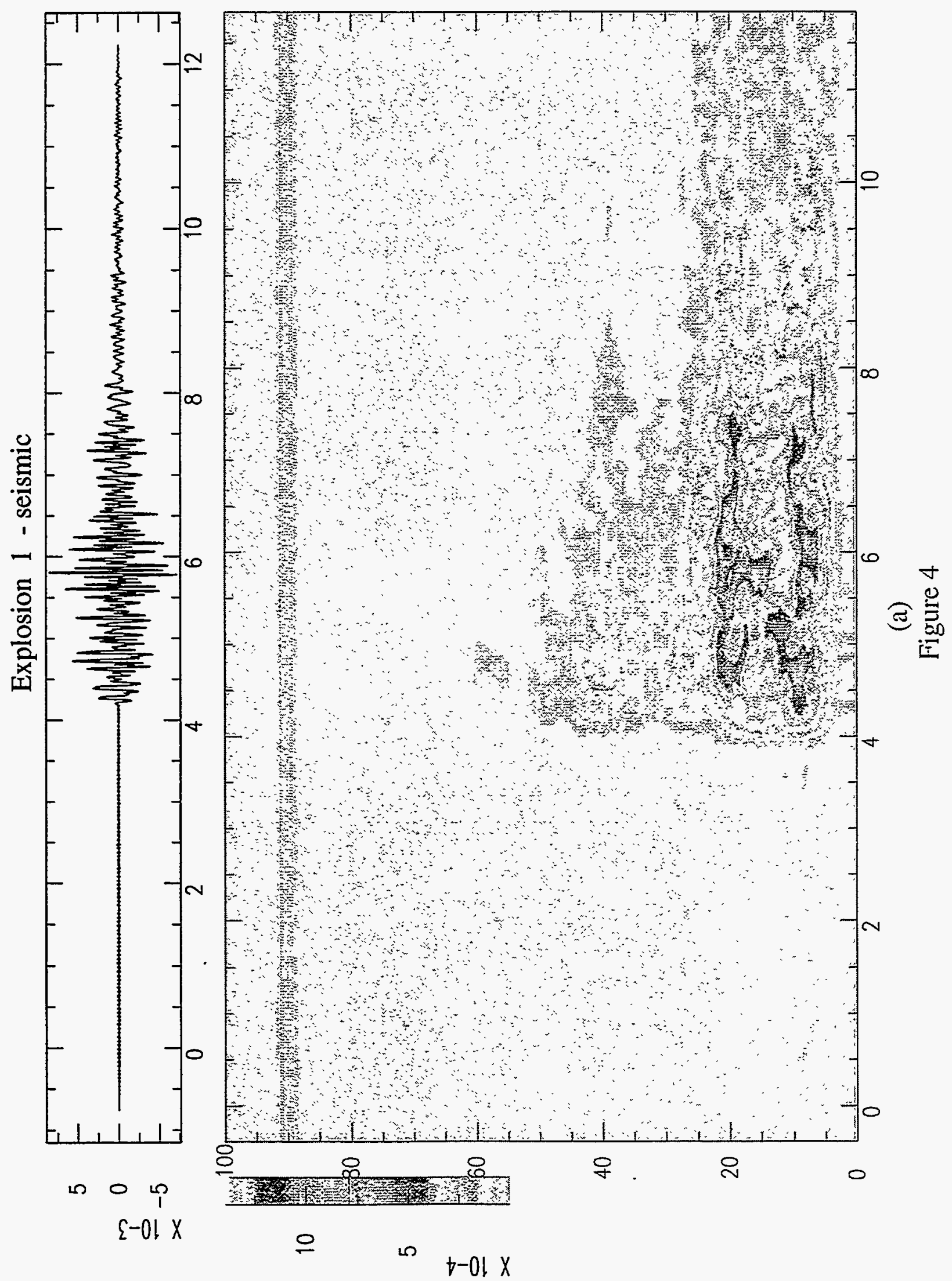




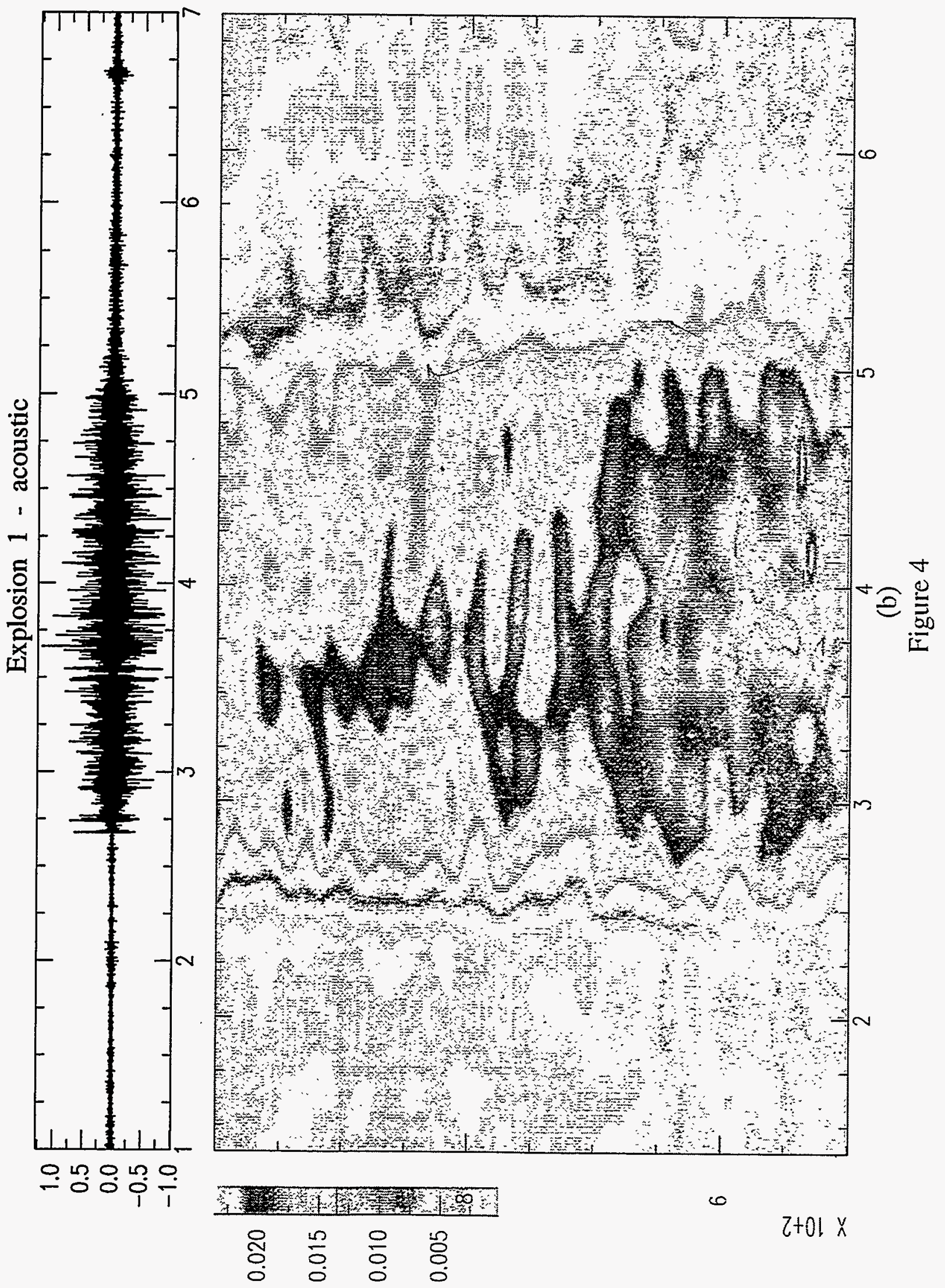


Explosion 2 - seismic
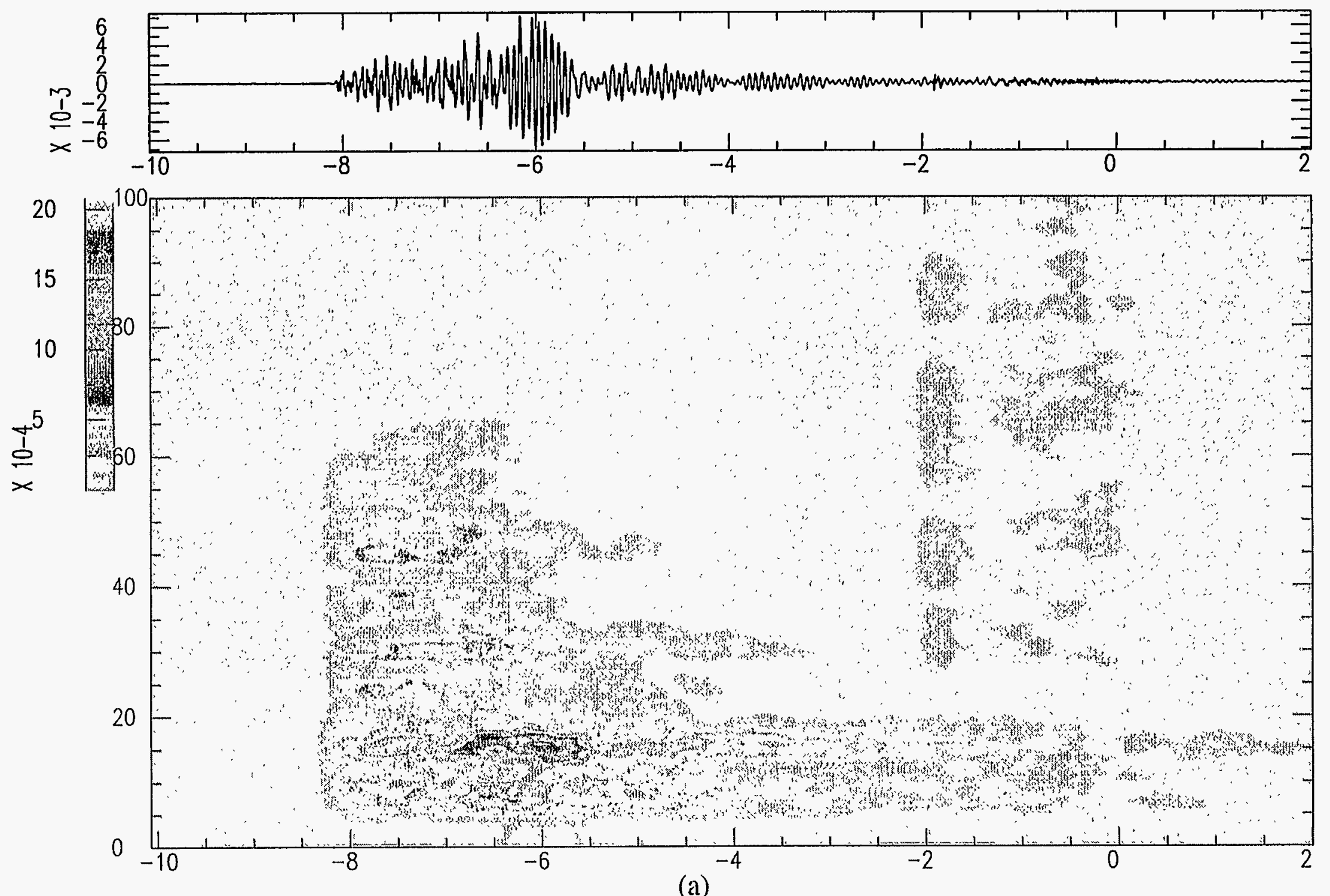

Figure 5 


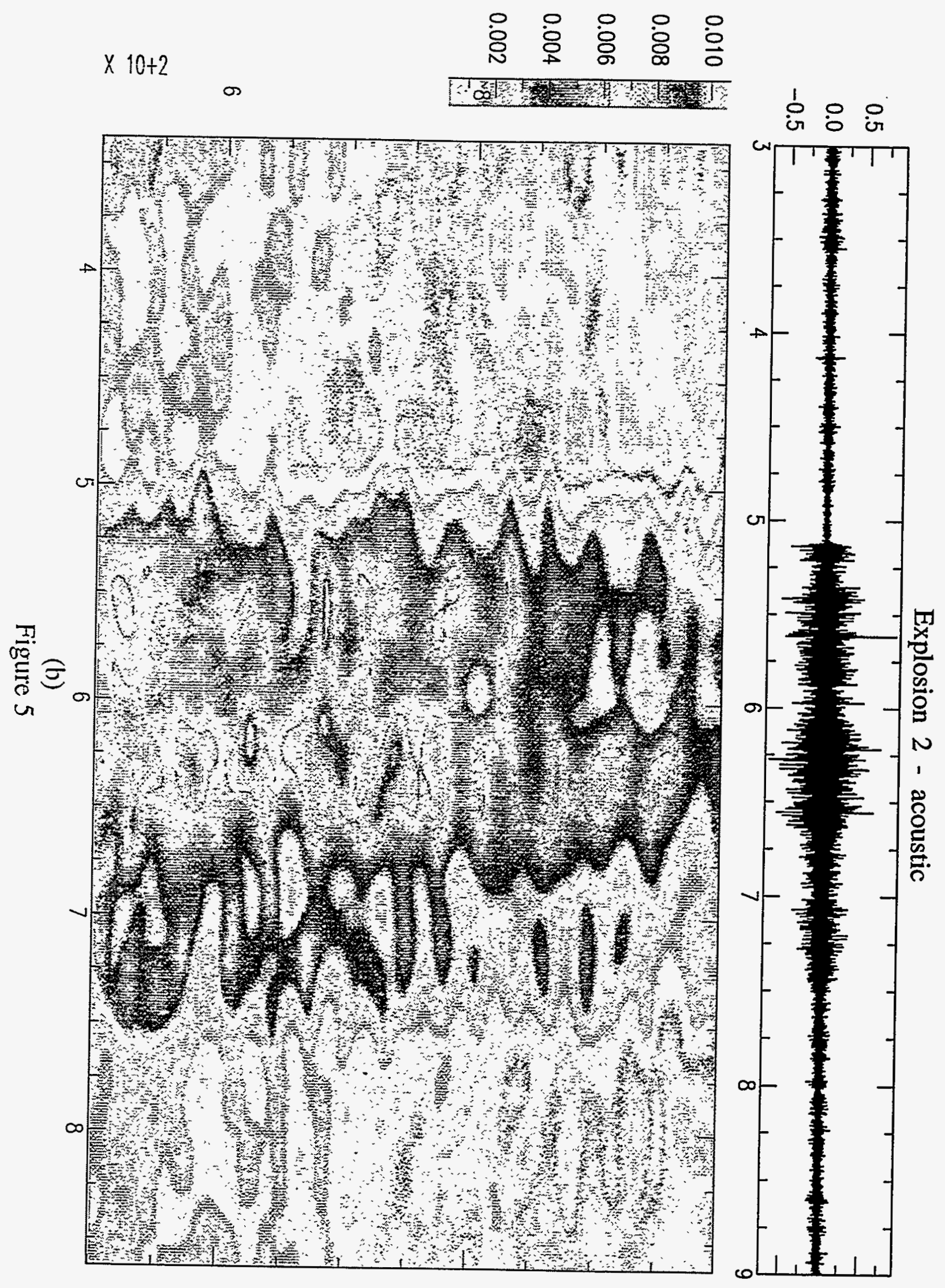



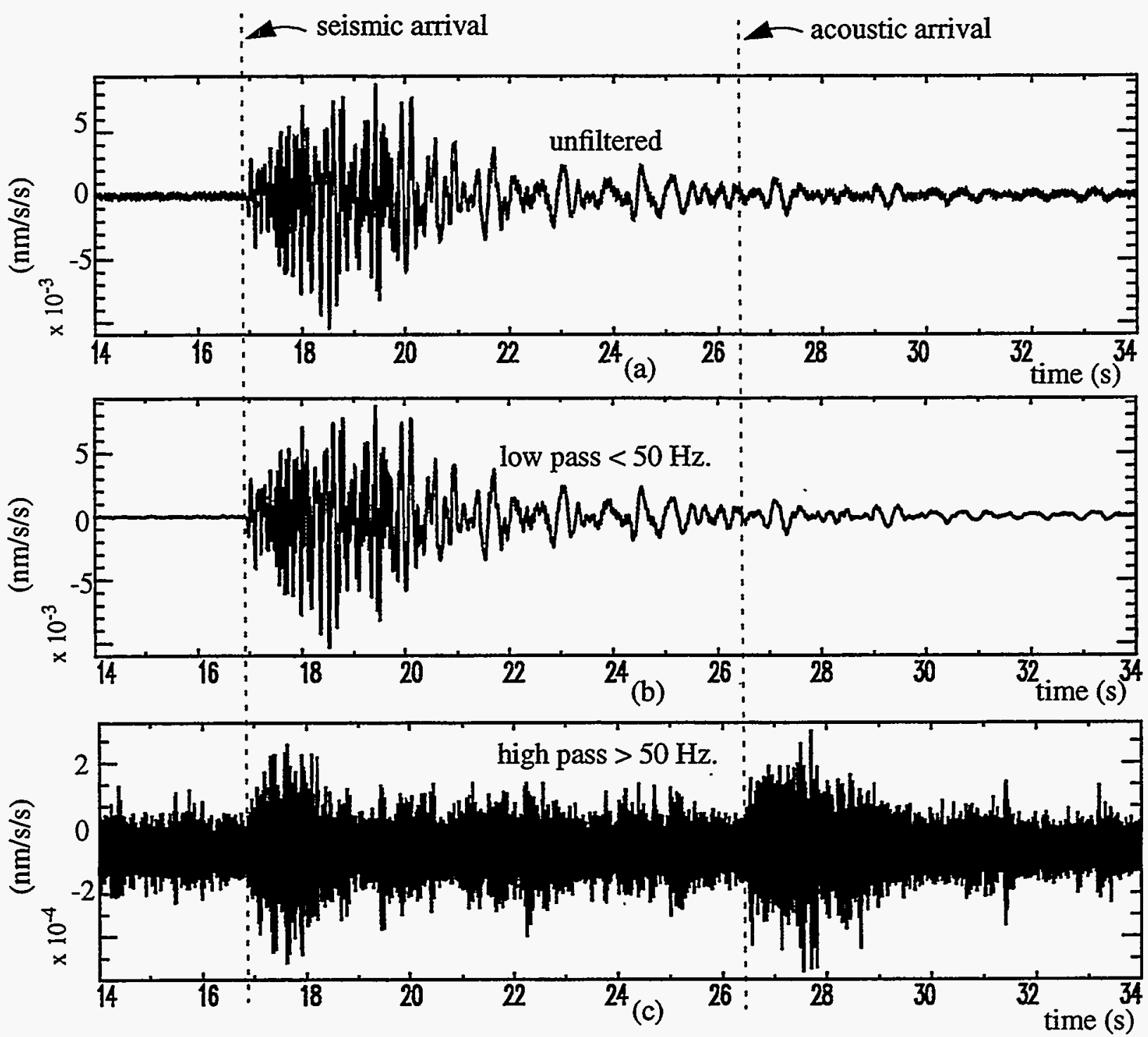

Figure 6 


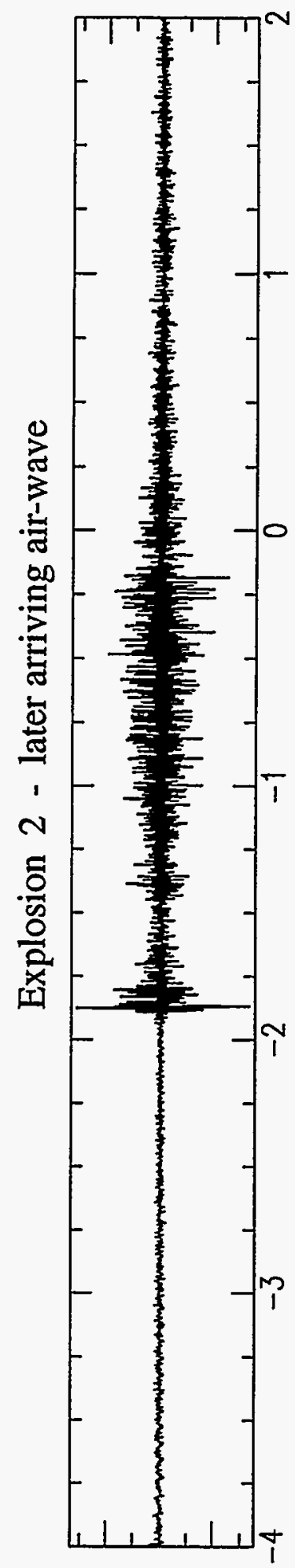

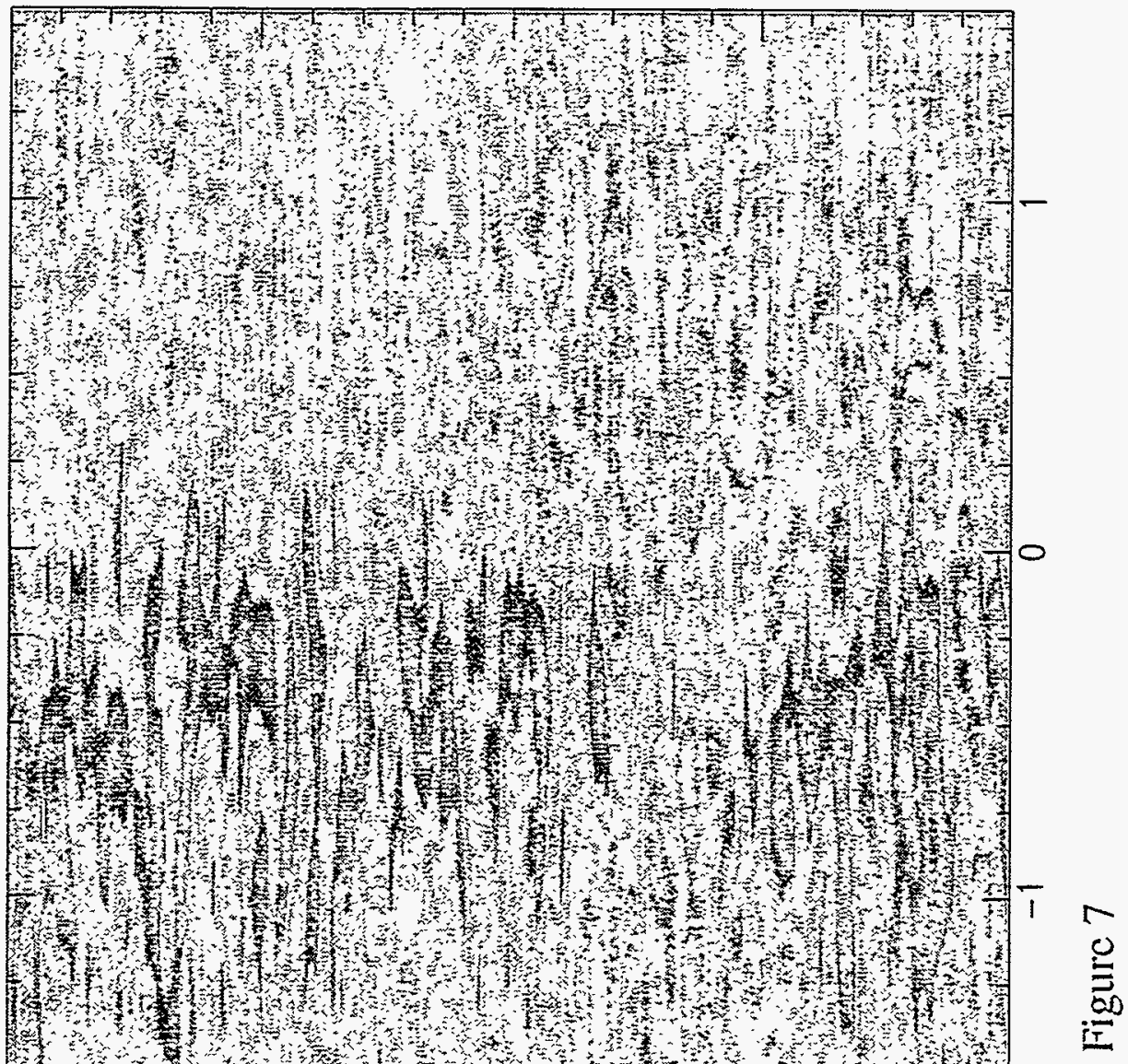

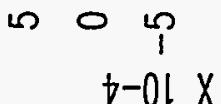

(2)

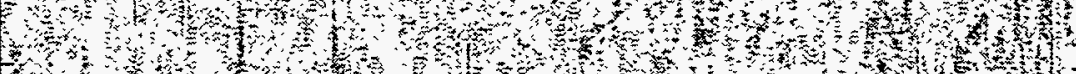

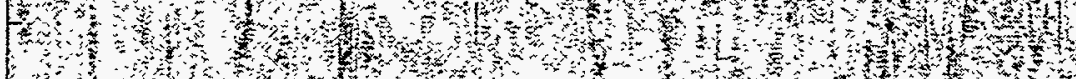

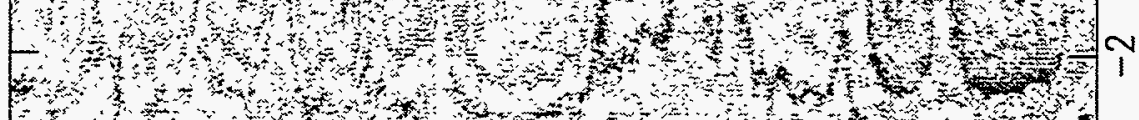

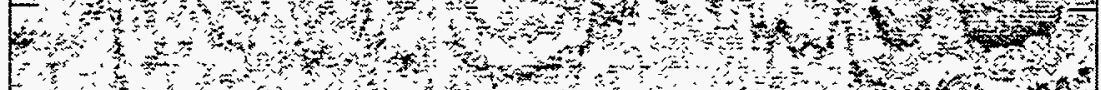

(a)

Fo

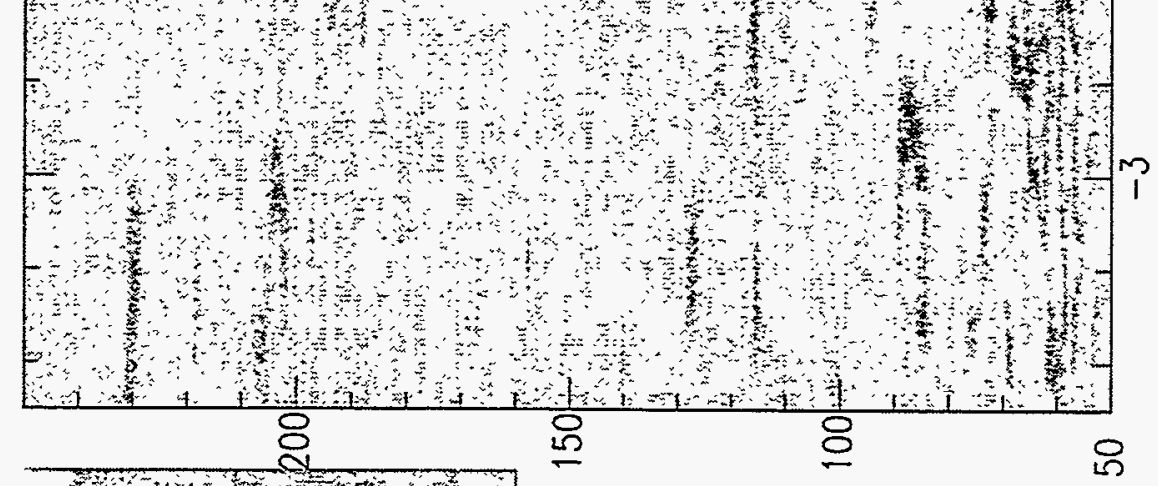

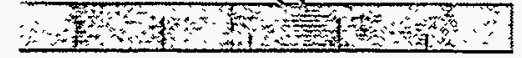

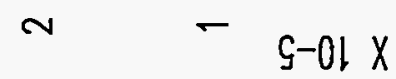




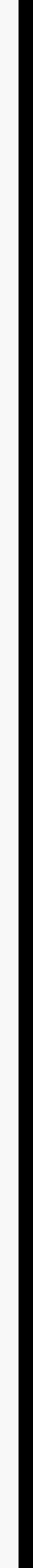



Technical Information Department - Lawrence Livermore National Laboratory University of California - Livermore, California 94551 